\title{
Tratamiento por vía transuretral de la hematuria masiva postadenomectomía retropúbica
}

\author{
Gaya Sopena JMª , Arce Gil J, Gausa Gascón L, Montlleó González M, Salvador Bayarri J, \\ Villavicencio Mavrich $\mathrm{H}$.
}

Servicio de Urología. Fundació Puigvert. Barcelona.

Actas Urol Esp. 2007;31(7):743-745

\begin{abstract}
RESUMEN
TRATAMIENTO POR VÍA TRANSURETRAL DE LA HEMATURIA MASIVA POSTADENOMECTOMÍA RETROPÚBICA

Introducción y objetivos: Evaluar la frecuencia de reintervención por hematuria postadenomectomía en nuestro centro al igual que la efectividad y los posibles efectos secundarios del abordaje transuretral como tratamiento resolutivo en el postoperatorio inmediato.

Material y métodos: Se analizaron retrospectivamente 540 adenomectomías retropúbicas realizadas en el periodo 1998-2005. Se evaluó la efectividad, el tiempo quirúrgico medio de reintervención y las complicaciones en los casos en que se realizó la revisión endoscópica.

Resultados: En un 2,5\% del total de adenomectomías retropúbicas fue necesaria por hematuria la revisión endoscópica. En todos estos casos se logró por esta vía el control hemostático. El tiempo quirúrgico medio fue de 37 minutos y los efectos secundarios observados insignificantes.

Conclusiones: La vía transuretral es una técnica simple y eficaz en el tratamiento de la hematuria masiva postadenomectomía retropúbica. El tiempo quirúrgico empleado es aceptable y las complicaciones observadas a largo plazo son mínimas y no pueden atribuirse únicamente a dicha técnica.

Palabras clave: Hematuria. Adenomectomía retropúbica. Reintervención. Transuretral.
\end{abstract}

\begin{abstract}
TRANSURETHRAL TREATMENT OF MASSIVE HEMATURIA POST RETROPUBIC ADENOMECTOMY

Introduction and objectives: to evaluate the frequency of reoperation caused by massive hematuria in the postoperation of open prostatectomy in benign prostatic hyperplasia (BHP) at our hospital. At the same time, we also want to evaluate the effectiveness and possible secondary effects of using transurethral approach to solve this surgical complications.

Material and Methods: we analyzed retrospectively 540 open surgeries in benign prostatic hyperplasia, carried out from 1998 to 2005. We evaluated effectiveness, average surgery time and complications in case of endoscopic review.

Results: a reoperation was necessary in $2.5 \%$ of all 540 cases. In all the cases reoperated, hemorrhage was controlled using transurethral approach. Average surgery time was 37 minutes and secondary effects observed were not important.

Conclusions: transurethral approach is a simple and effective technique in the treatment of massive hematuria after open prostatectomy in BPH. Surgery time spent is acceptable, and early and delayed complications observed have been few and cannot, in our opinion, be imputed only to this technique.
\end{abstract}

Keywords: Hematuria. Open prostatectomy. Reoperate. Transhuretral. 
$\mathrm{L}^{2}$ a necesidad de revisión hemostática por hematuria masiva en el postoperatorio inmediato de una adenomectomía retropúbica es una complicación poco frecuente, pero que sucede entre el 0,5 y el 3\% de los $\operatorname{casos}^{1,2}$.

A veces la reapertura del lecho quirúrgico puede ser compleja y en ocasiones poco efectiva por la dificultad en la localización del sangrado, terminando ocasionalmente con la realización de taponamientos y cerclajes en la celda prostática. Estas maniobras se ha visto que conllevan complicaciones tardias, como la esclerosis del cuello cervical, o inmediatas como es el atrapamiento ureteral ${ }^{3,4,5}$.

Algunos autores hablan de la vía transuretral para la revisión hemostática ${ }^{3}$, pero es poca la referencia en la literatura sobre el uso de esta vía para el tratamiento resolutivo de esta complicación postquirúrgica.

\section{OBJETIVOS}

Los objetivos que nos planteamos en nuestra revisión fueron:

1. Evaluar la frecuencia de reintervención por hematuria postadenomectomía retropúbica en nuestro centro.

2. Evaluar el tiempo medio empleado en la revisión endoscópica y compararlo con el tiempo medio empleado en la reapertura del lecho quirúrgico (vía abierta), de nuestra serie histórica.

3. Evaluar la efectividad, y los posibles efectos secundarios derivados del uso del abordaje transuretral como tratamiento resolutivo de la hematuria masiva en el postoperatorio inmediato de la adenomectomía.

\section{MATERIAL Y MÉTODOS}

Analizamos 540 adenomectomías retropúbicas realizadas entre los años 1998 y 2005, la técnica usada en todas ellas fue la de Millin modificada ${ }^{6,7}$.

En el postoperatorio inmediato se reintervinieron todos aquellos pacientes que presentaban hematuria masiva que no cedía con las maniobras habituales y cumplian criterios de reintervención (inestabilidad hemodinámica, necesidad de transfusión). En todos estos casos la reintervención fue vía endoscópica.

La técnica empleada consiste en introducir un resector doble vaina $26 \mathrm{Fr}$ bajo visión directa. En un primer tiempo se evacuan los coágulos adhe- ridos a la celda prostática con el asa de resección y en un segundo momento con mejor visión se buscan vasos sangrantes y se coagulan con el electrodo de bola $\mathrm{u}$ asa.

Se evaluaron el número de reintervenciones necesarias del total de 540 adenomectomías retropúbicas realizadas en nuestro centro en estos siete años.

Una vez finalizada la reintervención se valoró la efectividad de ésta y el tiempo medio empleado para la revisión hemostática.

En consultas externas se hizo un seguimiento medio de cuatro años de los pacientes reintervenidos con dicha técnica para valorar las posibles complicaciones o efectos secundarios que pudieran aparecer.

\section{RESULTADOS}

Fueron necesarias 14 reintervenciones, lo que supone un 2,5\% del total de adenomectomías retropúbicas realizadas en nuestro centro en siete años.

En el 100\% de los casos (14 de 14) la complicación postquirúrgica inmediata fue resuelta por vía endoscópica, no siendo necesaria la cirugia abierta.

Se evidenció con frecuencia como origen del sangrado vasos a nivel de ápex prostático y cara anterior, puntos de difícil accesibilidad por vía abierta y fácilmente accesibles con el resector. En ocasiones no se consiguió objetivar un único punto sangrante, siendo necesaria la coagulación amplia de la celda prostática. La celda prostática presentaba una superficie regular, tras la adenomectomía, deslizándose fácilmente el electrodo de bola.

El tiempo medio empleado en la revisión hemostástica transuretral fue de 37 minutos con un rango (20-56) minutos. El tiempo medio empleado en las últimas 14 revisiones con reapertura del lecho quirúrgico en nuestro centro, teniendo estas lugar antes del año 1998 fue de 54 minutos con un rango (36-120) minutos.

Las complicaciones observadas fueron: 2 estenosis uretra, 1 incontinencia (por imperiosidad demostrada en estudio urodinámico), 2 infecciones de orina no complicadas.

\section{CONCLUSIONES}

- La vía transuretral es una técnica simple y eficaz en el $100 \%$ de los casos para el tratamiento de la hematuria masiva postadenomectomía retropúbica, que no cede con las maniobras habituales. 
- El tiempo medio empleado es muy aceptable y menor al que necesitábamos cuando resolvíamos esta complicación vía abierta con reapertura del lecho quirúrgico.

- Las complicaciones observadas a largo plazo son mínimas y además no pueden atribuirse únicamente a dicha técnica ${ }^{8,9}$.

\section{REFERENCIAS}

1. Serretta V, Morgia G, Fondacaro L, Curto G, Lo bianco A, Pirritano D, et al. Open prostatectomy for benign prostatic enlargement in southern Europe in the late 1990s: a contemporary series of 1800 interventions. Urology. 2002 Oct;60(4):623-627.

2. Roos NP, Wennberg JE, Malenka DJ, Fisher ES, McPherson K, Andersen TF, et al. Mortality and reoperation after open and transurethral resection of the prostate for benign prostatic hyperplasia. N Engl J Med. 1989 Apr 27;320(17):1120-1124.

3. Cirugía abierta de la hipertrofia benigna de la próstata. B Dore, J Irani. EMC urología. E-41-275.

4. Lezrek M, Ameur A, Renteria JM, El Alj HA, Beddouch A. Modifided Modified Denis technique: a simple solution for maximal hemostasis in suprapubic prostatectomy. Urology. 2003 May;61(5):951-955.
5. Mebust WK, Holtgrewe HL, Cockett ATK, Peters PC. Transurethral prostatectomy: immediate and postoperative complications. Acoperative study of 13 partcipating institutions evaluating 3.855 patients. J Urol, 141: 243-247, 1989.

6. Millin T. Retropubic prostatectomy: A new extravesical technique. Report on 20 cases. J Urol. 2002 Feb;167(2 Pt 2):9769; discussion 980.

7. Walsh PC, Oesterling JE. Improved hemostasis during simple retropubic prostatectomy. J Urol. 1990 Jun;143(6): 12031204.

8. Fourcade R, Lanson Y, Teillac P. Results of the surgical treatment of benign hypertrophy of the prostate. Prog Urol. 1993 Oct;3(5):823-915.

9. Tubaro A, Carter S, Ahmad H, Vicentini C, Miano L. A prospective study of the safety and efficacy of suprapubic transvesical prostatectomy in patients with benign prostatic hiperplasia. J Urol 2001; 166: 172-176.

Correspondencia autor: Dr. J.M ${ }^{\mathrm{a}}$ Gaya Sopena

Servicio de Urología.Fundación Puigvert

Cartagena, 340-350 - 08025 Barcelona. Telf.: 934169700

E-mail autor: 36178jgs@comb.es

Información artículo: Original

Trabajo recibido: marzo 2007

Trabajo aceptado: abril 2007 